\title{
Fast Impedance Spectroscopy Method for Insulating Layers with Very High Impedance
}

\author{
P. Schauer* \\ Department of Physics, Faculty of Civil Engineering, BUT, Zizkova 17, 60200 Brno, Czech Republic \\ * corresponding author: schauer@dp.fce.vutbr.cz
}

DOI: $10.2478 / \mathrm{v} 10158-010-0005-1$

\begin{abstract}
This paper presents the fast impedance spectroscopy method for objects with a very high impedance $\left|Z_{\mathrm{x}}\right| \geq 1 \mathrm{G} \Omega$ modeled by $\mathrm{RC}$ networks. The method is suitable for predicting the lifetime and reliability of insulating materials. The fast impedance spectroscopy method was tested on insulating layers which were part of the system metalinsulator-metal (MIM). The method is based on measurements of the power loss and phase shift in the AC voltage divider, which consists of the measured system MIM and of the known impedance. Computer controlled instruments measure the gain and phase shift between the two outputs of alternating electrical circuit and a computer program calculates the parameters of the impedance spectroscopy from these values. The output is the graphical and tabular impedance characteristics of the MIM system, namely: the dielectric loss of the insulating film, imaginary part of impedance, loss factor, parallel resistance $R_{p}$ and the capacity $C_{p}$ of the MIM system, all frequency dependent. Frequency characteristics provide an analysis of loss mechanisms that may affect the quality and reliability of the insulating layers.
\end{abstract}

KEY WORDS: computer program, impedance spectroscopy, loss factor, insulating layers.

\section{INTRODUCTION}

Insulators and dielectrics have an irreplaceable role in electrical engineering and their presence is a necessary condition for the function of almost every electrical device. High-voltage electrical insulators are of special importance, where their quality affects the life and reliability of the whole equipment. The quality of the insulation material is limited by the working electric field intensity. Insulators are used in insulated wires and cables, in electrical machines and devices, and transformers and electric motors. High-voltage electrical engineering uses capacitors, as well as printed circuit boards. The most important material characteristics of insulators are conductivity, permittivity, dielectric loss factor and electrical strength. In most technical applications the dielectric losses are a scourge, causing dielectric heating and subsequent deterioration of its functional characteristics and reliability, especially in power applications.

Most recently a new method of dynamic monitoring of insulators and dielectrics was developed, known as impedance spectroscopy. It monitors the complex impedance dependence on frequency. The important dependences of impedance spectroscopy are mainly: 
the frequency dependence of the real part of impedance, the frequency dependence of resistance, and, mainly, the frequency dependence of loss-factor, which is independent of the geometry of an insulator, which is a parameter of material only.

\section{THEORY}

From a physical point of view we can distinguish three main processes that give rise to dielectric losses:

1. The main process causing the dielectric loss is the process of electrical conductivity. This type of the dielectric loss is called conductivity losses. Conductivity losses appear in all types of dielectrics and insulators. Most apply at higher temperatures.

2. The second dielectric losses are polarization losses. These losses are usually only found in a certain temperature range where the loss polarization applies. They are already noticeable at less than microwave frequencies.

3. Ionization losses are added to the above processes in strong electric fields. Ionization losses exist because of dielectric ionization.

The processes of conductivity losses, polarization losses, and ionization losses are independent in the analysis of dielectric losses and thus, according to the additive principle, they are the sum of the individual components.

Dielectric losses are defined by the power relationship

$$
P=U I \cos \varphi
$$

Here $U, I$ are the voltage RMS and current RMS respectively, $\varphi$ is the phase shift between current and voltage. It is not convenient to express dielectric loss directly because it depends on the geometry of the material. It is better to introduce a loss factor, which is a material parameter, independent of geometry. The dielectric losses are described by the relationship

$$
\tan \delta=\frac{P}{U^{2} \omega C}
$$

$C$ is the capacity and $\omega$ is the frequency. In non-polar dielectrics, the conductivity losses only occur depending on the frequency of the electric field, at $P=$ const. Consequently, according to equation (2), loss factor must be hyperbolic with increasing frequency, $\tan \delta \sim 1 / f$. Model behavior is shown in Figure 1, in logarithmic scales, for three different values of $P / U^{2}$ and for the value of capacity $C=50 \mathrm{pF}$.

As Figure 1 shows, the conductivity loss component of the loss-factor added to the polarization component in polar dielectrics. As such, the polarization component of the loss-factor reaches a peak when the reverse frequency value equals the time constant of the relaxation polarization effect $\omega=1 / \tau$. The loss-factor has a similar dependence as a resonance curve. 
Curve 4 in Figure 1 is modeled by the equation

$$
\tan \delta=\frac{a}{\sqrt{b^{2}+\left(c \omega-\frac{1}{d \omega}\right)^{2}}},
$$

where constants $c, d$ satisfy the condition of frequency maximum of polarization loss-factor

$$
\omega_{\mathrm{m}}=\frac{1}{\sqrt{c d}}
$$

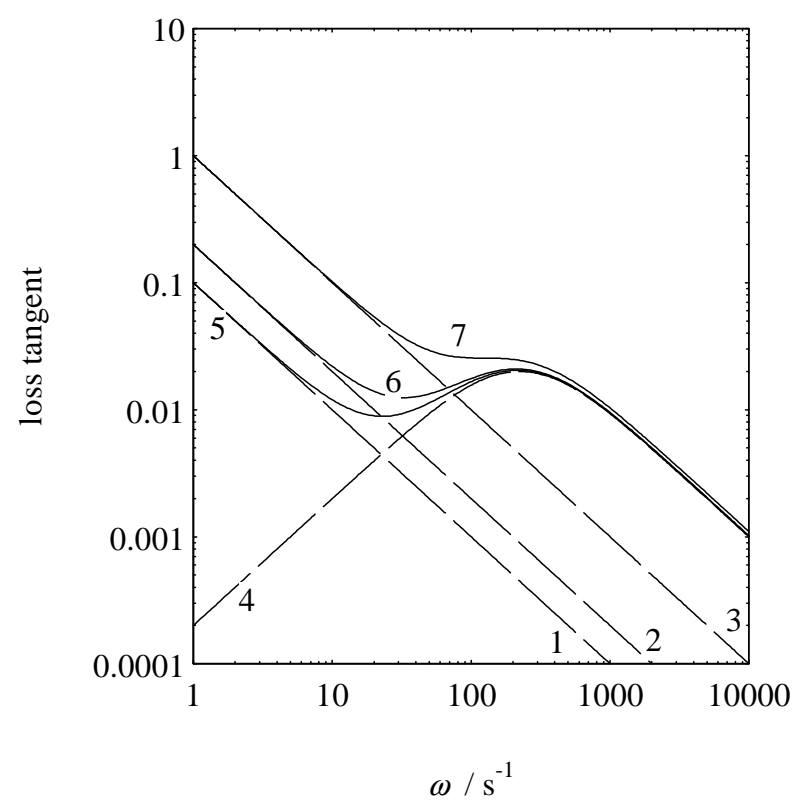

Figure 1: Model of the loss-factor dependences versus electric field frequency, both in logarithmic scales. Dashed curves $1,2,3$ of type $1 / f$ correspond to conductivity losses. Conductivity loss-factor is modeled according to equation (2) for three different values of $P / U^{2}$ and for the value of capacity $C=50 \mathrm{pF}$. Dashed curve numbered 4 (with a maximum) corresponds to polarization losses. Full curves 5, 6, 7 are the sum of both components.

The total loss factor is the sum of the above mentioned two loss mechanisms. Local excess of the curve $\tan \delta=f(\omega)$ over the hyperbole indicates the presence of dielectric polarization, which is a negative indicator for the reliability and quality.

Another view of the loss mechanisms can be seen if we plot dielectric losses $P$, depending on the frequency, for the same model parameters as in Figure 1. The results are shown in Figure 2. Curves are obtained by multiplying the loss-factor by $U^{2} \omega C$, see equation (2). Numbering of curves in Figure 2 corresponds to numbering in Figure 1. We see that while the conductivity losses component (curves $1,2,3$ ) is frequency independent and applies even at low frequencies, polarization losses (curve 4) do not apply for the low frequencies, because it is very small. However from a certain frequency, when sharply increasing, 
polarization losses apply, and for sufficiently high frequency polarization losses are, like conductivity losses, constant.

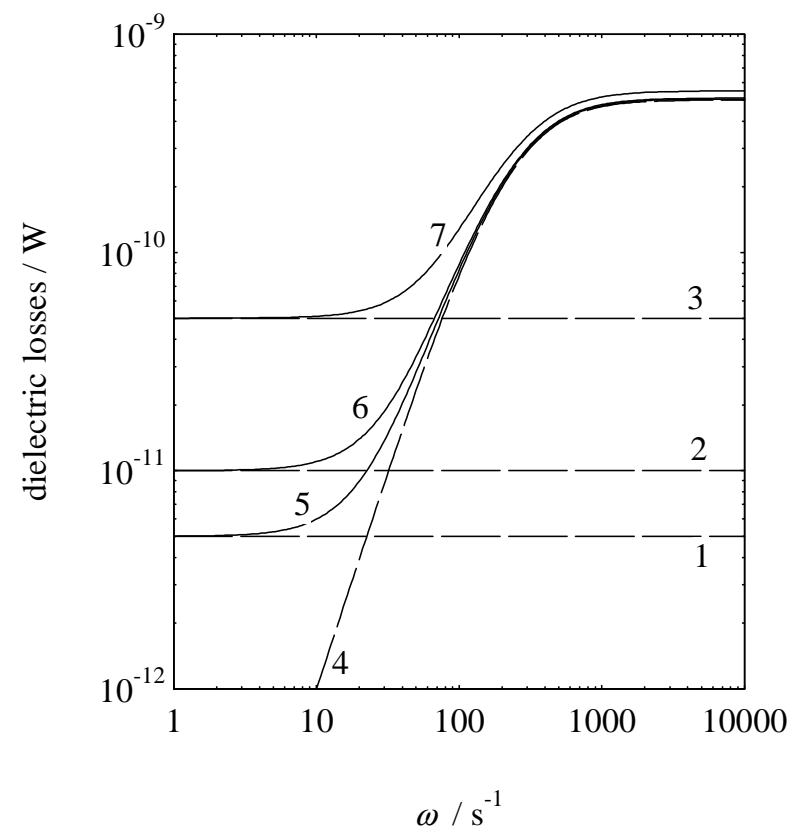

Figure 2: Dielectric losses versus frequency for the same model parameters as in Figure 1. We multiplied the loss-factor by $U^{2} \omega C$ value using equation (2), where $U^{2} C=50.10^{-12} \mathrm{~J}$. Numbering of curves corresponds to those in Figure 1.

While polar dielectrics have a much higher relative permittivity compared to the non-polar, its value depends on the temperature and electric field frequency. Thus they have a much higher frequency-dependent dielectric loss and much higher conductivity than the non-polar, which also depends on temperature. Humidity has a considerable effect. With the exception of lower temperatures, polar dielectrics have significantly less electrical resistance than un-polar. All these features indicate that the study of the presence of the polarization phenomena in dielectrics is important diagnostic information about the quality and reliability of a dielectric insulator.

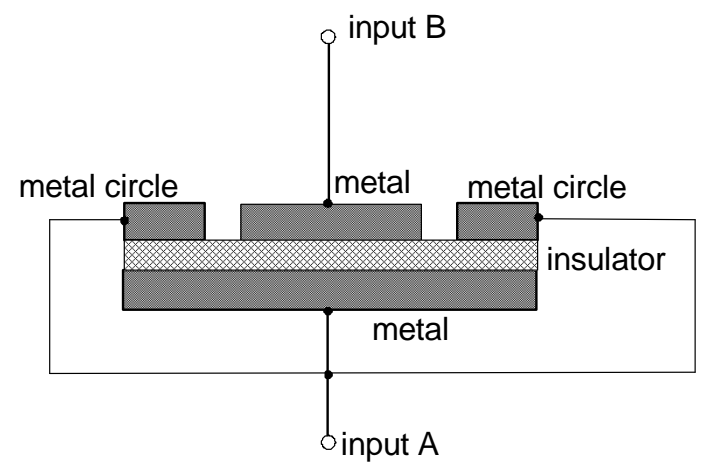

Figure 3: Insulating film is placed in a metal holder. 


\section{EXPERIMENTAL SETUP}

The detailed setup of the MIM system is shown in Figure 3. A metal holder consists of a bottom circular electrode attached to the input A of the gain-phase meter (Figure 4) and opposite top circular electrode is connected to input B of the gain-phase meter. The ring around the top metal electrode is electrically connected with the bottom electrode. The holder was made of brass.

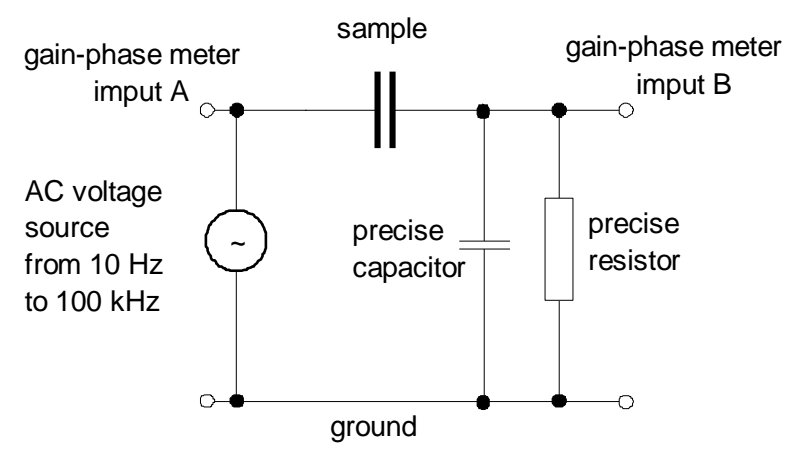

Figure 4: Detailed schema of blocks AC VS and DUT from the Figure 5. Exact resistance $1.07 \mathrm{M} \Omega$ consists of input $\mathrm{B}$ resistance, capacitor consists of $\mathrm{B}$ input capacity 112 pF.

Frequency dependences of physical quantities evaluated in the impedance spectroscopy were detected from the ratio of voltage and the phase shift of inputs A, B of the gain-phase meter (Figure 4). We used the instrument Hewlett Packard HP3575A, which operates in the frequency from $1 \mathrm{~Hz}$ to $13 \mathrm{MHz}$. The circuit was fed with a signal generator connected to input A.

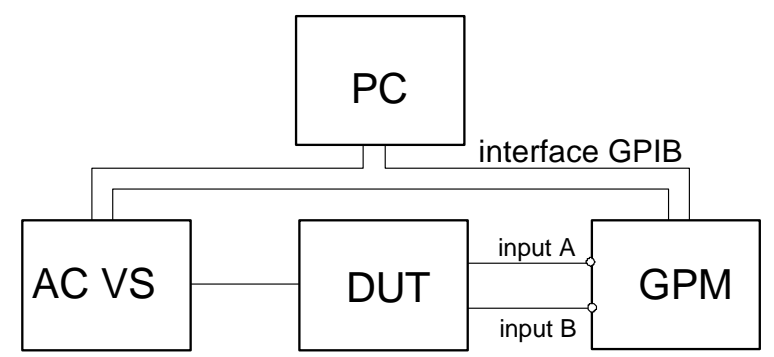

Figure 5: Block diagram of the measuring arrangement. AC VS is alternating sinusoidal current supply, DUT is MIM device under test, the GPM is gain phase meter and the $\mathrm{PC}$ is the computer.

Figure 5 shows the block diagram of the setup, where AC VS is the sinusoidal signal generator working in the frequency range from $1 \mathrm{~Hz}$ to $13 \mathrm{MHz}$, DUT is tested MIM system (device under test), the GPM is the gain phase meter with two inputs and the PC is the computer. 
Gain $L$ and phase shift $\varphi$ between the inputs A, B were measured. Gain is defined as

$$
L=20 \log K
$$

where $K=\mathrm{U}_{\mathrm{B}} / U_{\mathrm{A}}$. It seems that the equivalent parallel $\mathrm{RC}$ circuit corresponds best to the MIM systems in real conditions. Therefore we replaced the tested MIM system with a RC combination $R_{\mathrm{p}}=R_{\mathrm{x}}$ and $C_{\mathrm{p}}=C_{\mathrm{x}}$. Under these conditions, the phase of the parallel impedance of resistance $R_{\mathrm{B}}$ and input capacitor $C_{\mathrm{B}}$ (of GP meter) is

$$
\varphi_{B}=-\arctan \omega R_{B} C_{B},
$$

Impedance of parallel combination of $R_{\mathrm{B}}, C_{\mathrm{B}}$ can be calculated as

$$
Z_{x}=\frac{Z_{B} \sin \varphi}{K-\sin \left(\varphi_{B}-\varphi_{x}\right)},
$$

Phase of $Z_{x}$ is

$$
\varphi_{x}=\varphi_{B}+\arctan \frac{\sin \varphi}{K-\cos \varphi} .
$$

Measured impedance $Z_{x}$ will have a value

$$
Z_{x}=\frac{Z_{B} \sin \varphi}{K-\sin \left(\varphi_{B}-\varphi_{x}\right)}
$$

Its real part is

$$
\operatorname{Re}\left(Z_{x}\right)=Z_{x} \cos \varphi_{x}
$$

and its imaginary part is

$$
\operatorname{Im}\left(Z_{x}\right)=Z_{x} \sin \varphi_{x}
$$

Hence loss factor of the test insulator, defined by the ratio of imaginary and real part of impedance $Z_{x}$ is

$$
\tan \delta_{x}=\frac{1}{\tan \varphi_{x}}
$$

the resistance of the parallel RC circuit of MIM system is

$$
R_{x}=\operatorname{Re}\left(Z_{x}\right)\left(1+\tan ^{2} \varphi_{x}\right)
$$

and its capacity is 


$$
C_{x}=-\frac{\tan \varphi_{x}}{\omega R_{x}} .
$$

\section{COMPUTER PROGRAM}

Our measuring program measures the mentioned parameters of the impedance spectroscopy using a computer with the GPIB bus and evaluates impedance characteristics of the height ohms samples, in accordance with equations (5) to (14). Program IMPEDANC was written in the language Turbo Pascal (Borland) for OS DOS. It applies object oriented programming for easy and efficient use. It is in the windows architecture, and steps are offered through the menu. The operator can use the mouse.

\subsection{Program description}

The main menu of the program IMPEDANC is in Figure 6. The menu allows choosing the main operations, such as head, measurement, numerical processing of the results and graphical processing of results. It is possible to return to any step whenever operator wants. The program has help that can be called either from the main menu or at any time during the measurement by pressing the F1 key.

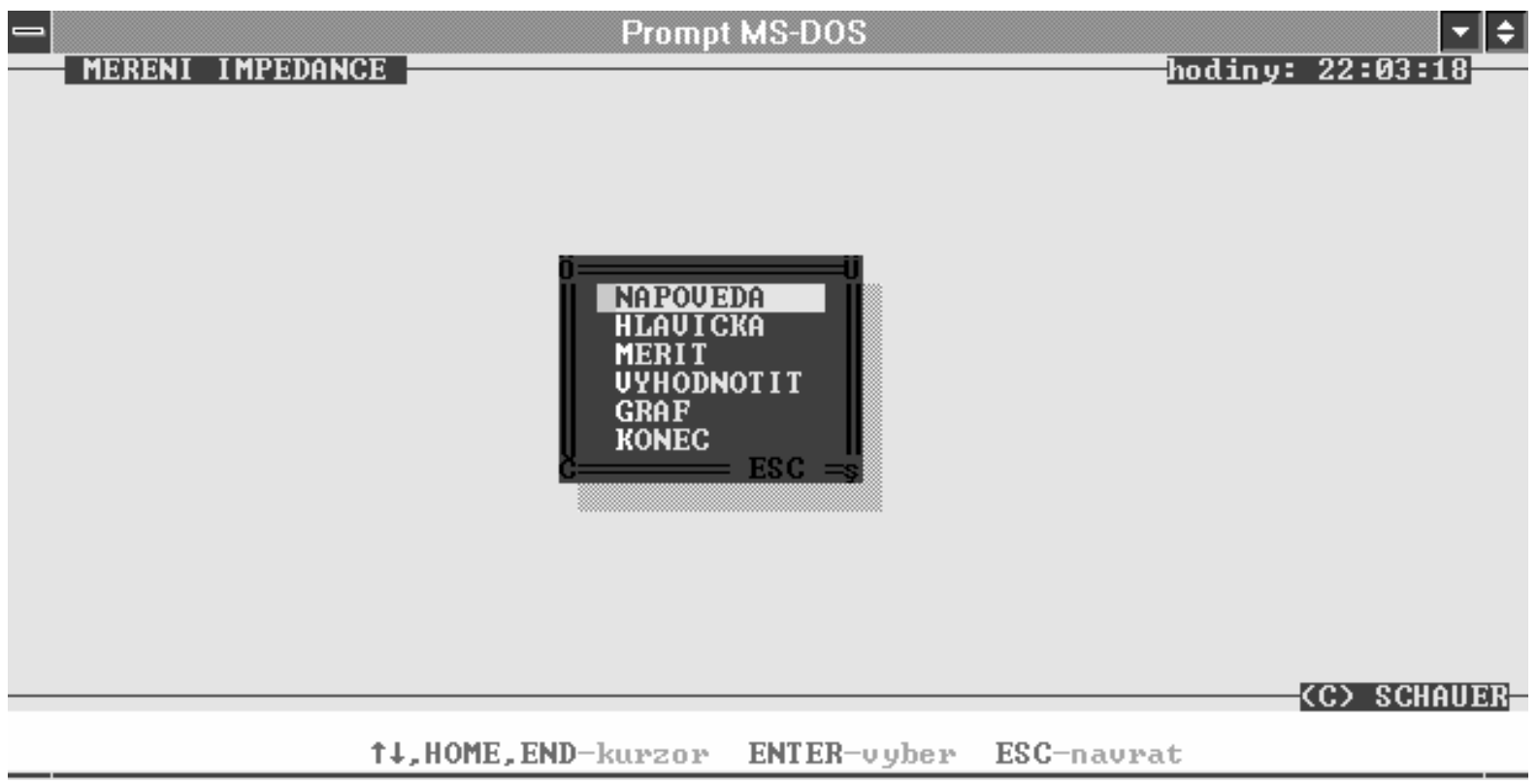

Figure 6: The main menu of the program impedanc.exe.

\subsubsection{Header creation}

Before we start the measurement we create a header from the main menu. Menu environment is shown in Figure 7. The header is pre-defined in the file IMPEDANC.INI. Item measure name may not remain empty, otherwise the completion of the header will be not allowed. 


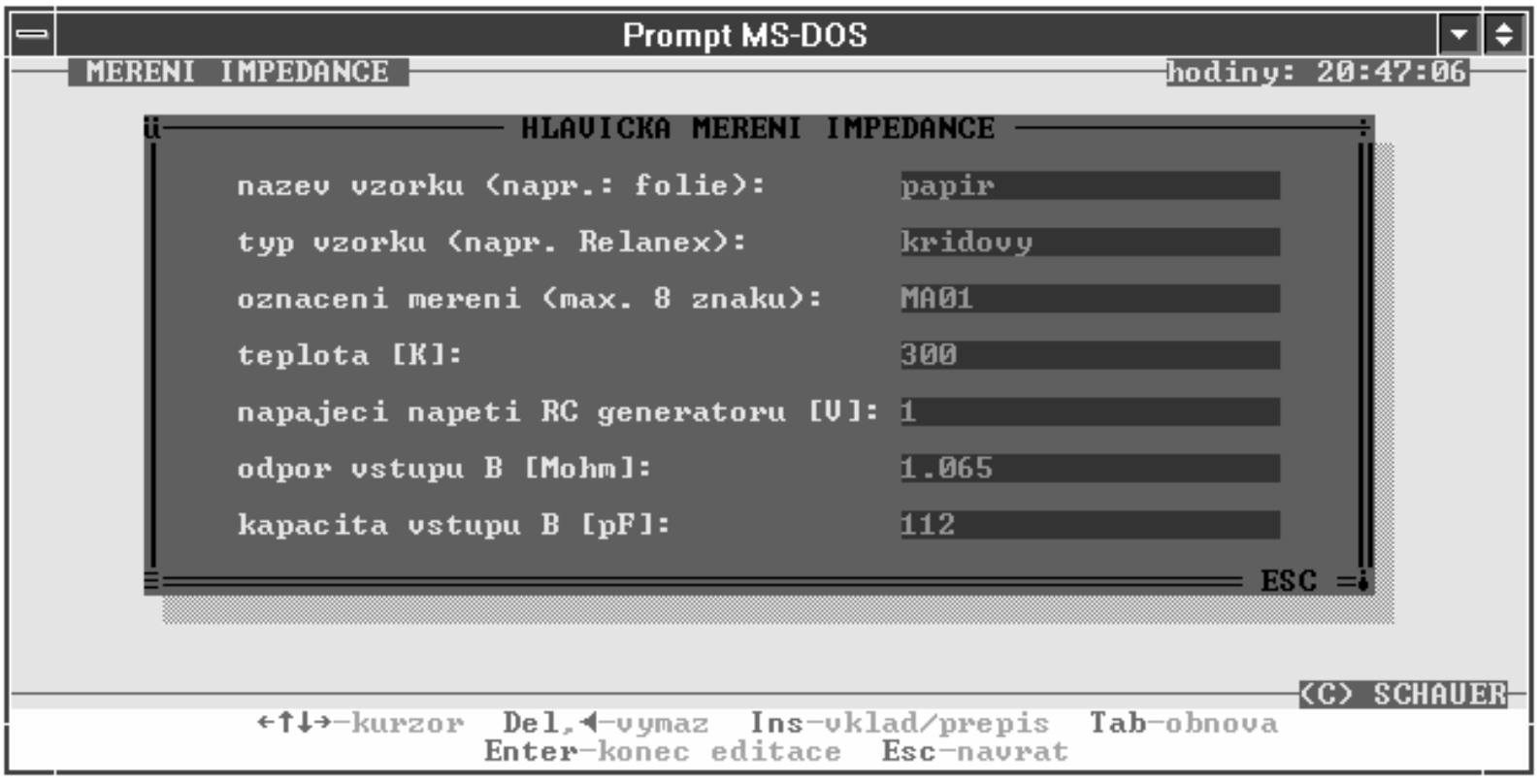

Figure 7: Items of measurement header are predefined in the file impedanc.ini and thus the measurement head is easy to fill in.

\subsubsection{Measurement}

Measurement starts by selecting 'Measure' from the main menu. The measurement results are stored in an output file with the same name as the measurement name in the header, but with the extension IMP. These files can be further processed by selecting 'Processing' in the main menu.

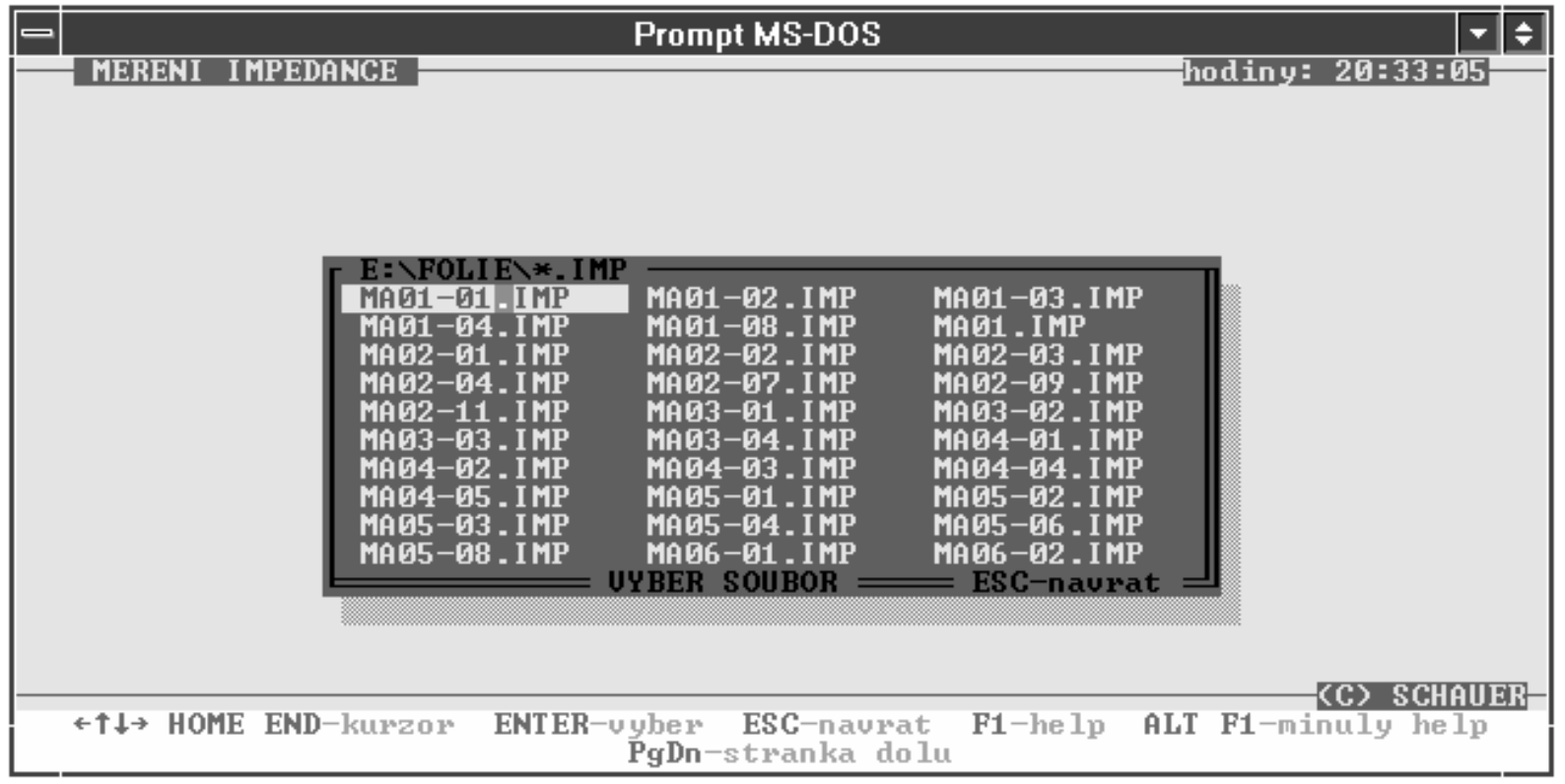

Figure 8: Clicking on the processing, the operator obtains a list of files with the extension IMP (files are the outputs from the measuring program - ready for further processing). 


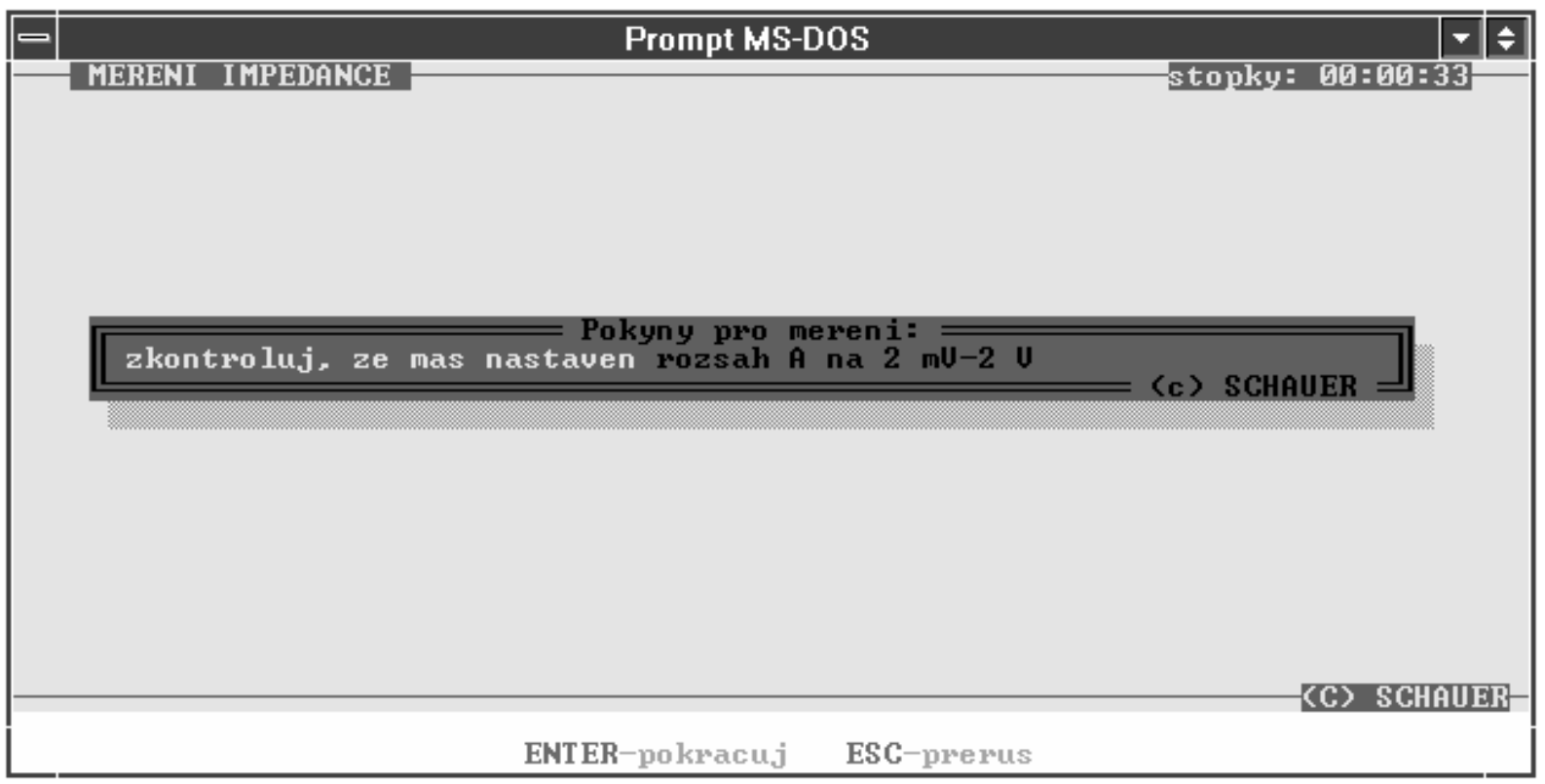

Figure 9: The operator is asked to control apparatus and instruments, during the measurement.

\subsubsection{Processing}

As shown in Figure 8, this operation offers a list of files with the extension IMP that are the output of our measurement program. These files are located in the working directory. We select one of the forms of evaluation. Then the process of impedance spectroscopy starts. Results are the set of tables and graphs $Z(f), \operatorname{Re} Z(f), \operatorname{Im} Z(f), \operatorname{tg} \delta(f), R_{p}(f)$, $C_{p}(f)$, that we discussed in the theoretical part of this contribution.

\section{CONCLUSION}

Impedance spectroscopy of insulating layers based on monitoring the impedance characteristics of metal-insulator-metal (MIM) structures is a relatively fast, non-destructive testing method suitable for predicting the lifetime and reliability of insulating materials. The published method here is based on measurements of gain and phase shift on the AC voltage divider, which consists of the measured system MIM and of the known impedance. The most appropriate examined parameter of the insulating film is frequency dependence of loss-factor, or the frequency dependence of dielectric loss $P$.

Polarization dielectric losses are an important criterion for testing the quality and reliability of dielectrics, especially in power applications.

\section{ACKNOWLEDGMENTS}

This paper was supported by the Grant Agency of the Czech Republic, project number 102/94/0858. 


\section{REFERENCES}

Kusak, I.: 2009. Impedance Spectroscopy of Ceramic (Plain) Roofing Tiles, International Interdisciplinary Technical Conference of Young Scientist Intertech 2009, ISBN Politechnika Poznan, Poznan, Poland.

Lunak, M.: 2009. Non Destructive Testing of Cetris-Basic Wood-Cement Chipboards by Using Impedance Spectroscopy, The 10th International Conference of The Slovenian Society for Non-Destructive Testing "Application of Contemporary Non-Desructive Testing in Engineering", Slovinsko Ljubljana.

Schauer, P.: 2001. Transport and Noise properties of CdTe(Cl) Crystals. Microelectronics Reliability, vol. 41, iss. 3, pp. 431-436. 